\title{
Beyond the genomics blueprint: the 4th Human Variome Project Meeting, UNESCO, Paris, 2012
}

\author{
Maija R.J. Kohonen-Corish, MSc, PhD ${ }^{1,2}$, Timothy D. Smith, $\mathrm{BSc}^{3}$ and Helen M. Robinson, MSc, MPhil'; \\ on behalf of the delegates of the 4th Biennial Meeting of the Human Variome Project Consortium
}

The 4th Biennial Meeting of the Human Variome Project Consortium was held at the headquarters of the United Nations Educational, Scientific and Cultural Organization (UNESCO) in Paris, 11-15 June 2012. The Human Variome Project, a nongovernmental organization and an official partner of UNESCO, enables the routine collection, curation, interpretation, and sharing of information on all human genetic variation. This meeting was attended by more than 180 delegates from 39 countries and continued the theme of addressing issues of implementation in this unique project. The meeting was structured around the

At the completion of the Human Genome Project and the publication of the first high-quality sequence of the human genome almost 10 years ago, it was declared that the genomic era had begun. ${ }^{1}$ Francis Collins and his colleagues ${ }^{1}$ envisaged the future of genomics and predicted that this "blueprint for the genomic era" would bring a number of grand challenges at three levels, "genomics to biology, genomics to health, and genomics to society." The Human Variome Project (HVP) has been described as the "natural successor to the Human Genome Project." ${ }^{\text {The }}$ HVP addresses the challenges posed by genetic discoveries by enabling the collection, curation, interpretation, and sharing of information on all human genetic variations (http://www. humanvariomeproject.org/). This mission is summed up by its mantra, "sharing data-reducing disease." The HVP was offcially launched in Melbourne, Australia, in 2006, ${ }^{3,4}$ followed by biennial meetings. ${ }^{2,5}$

\section{MEETING OBJECTIVES}

The 4th Biennial Meeting of the Human Variome Project Consortium (HVP4) continued the theme of the previous meetings and addressed issues of implementation in this unique project. The HVP4 was held in Paris at the headquarters of the United Nations Educational, Scientific and Cultural Organization (UNESCO), 11-15 June 2012. It was co-organized by the HVP International Coordinating Office (Melbourne, Australia) and the International Basic Sciences Programme, the Natural Sciences Sector of UNESCO (Paris, France). It was attended by more than 180 delegates from 39 countries representing all the continents, bringing together researchers, journal editors, database managers and curators, clinical geneticists, and other health-care professionals. four main themes of the Human Variome Project strategic plan, "Project Roadmap 2012-2016": setting normative function, behaving ethically, sharing knowledge, and building capacity. During the meeting, the members held extensive discussions to formulate an action plan in the key areas of the Human Variome Project. The actions agreed on were promulgated at the Project's two Advisory Council and Scientific Advisory Committee postconference meetings.

Genet Med 2013:15(7):507-512

The objectives of the meeting were as follows: (i) to present the project's strategic plan for the next 5 years; (ii) to ensure progression of the objective of the HVP to embed the collection and sharing of genetic variation information into routine clinical practice; (iii) to present activities under way or planned toward the HVP objective; (iv) to integrate current activities where possible; (v) to establish collaborations and activities where the need exists; (vi) to gather experts in the strategies and software required together with those responsible for generating data; and (vii) to gather those who are already collecting mutations within their counties together with those who wish to establish a system, especially those from developing countries.

\section{THE HVP IS THE ONLY OFFICIAL PARTNER OF UNESCO IN GENETICS}

The meeting was officially opened by David Abraham, chairman of the HVP Board of Directors, and Richard Cotton, scientific director of the HVP, who outlined the major developments that have taken place since the last meeting and the growth of the consortium to more than 700 members from 69 countries. The HVP is now established as a legal entity, which allows it to enter into agreements and partnerships with governments and other organizations. Fifteen country nodes and 39 databases have been signed up, and China has joined as the first core member. Maciej Nalecz, director of the International Basic Sciences Programme at UNESCO, welcomed the meeting participants and expressed the strong commitment of UNESCO for the principles and mission of the HVP. UNESCO provides valuable support to the HVP through its experience in creating partnerships, capacity building, and fostering cooperation

${ }^{1}$ Cancer Research Program, Garvan Institute of Medical Research, St Darlinghurst, New South Wales, Australia; ${ }^{2}$ St Vincent's Clinical School, University of New South Wales, Sydney, New South Wales, Australia; ${ }^{3}$ Human Variome Project International Limited, Melbourne, Victoria, Australia. Correspondence: Timothy D. Smith (tim@variome.org) 
between centers of excellence. The HVP has worked closely with UNESCO since the project was established, and UNESCO recognized this close working relationship by endorsing the HVP as an official partner in 2011.

The meeting participants also paused to remember and pay tribute to pioneering geneticist David Rimoin, who passed away recently. David Rimoin was a true champion of HVP and the first chair of the project's International Scientific Advisory Committee (Mike Watson).

\section{CHINA ANNOUNCES A MAJOR GRANT-FUNDING PROGRAM FOR HVP MEMBERS}

A major milestone for the HVP was the partnership established with China, which was officially launched in Beijing in December 2011. ${ }^{6}$ In an exciting expansion of this partnership, Xitao Li, chairman of the HVP China Node, announced the HVP/China Country Development Program, which has allocated funds (US\$1 million) to support the growth of a viable, sustainable network of HVP Country Nodes. The funding agreement was formally signed at the meeting by Xitao Li and David Abraham. These funds are generously provided by China and are administered by the HVP. Applications are now sought from HVP members for projects that promote capacity building and enable the establishment of HVP nodes (http://short. variome.org/hvpccdp).

\section{ROADMAP TO THE FUTURE}

The HVP strategic plan for the next 5 years, Project Roadmap 2012-2016, was presented on the first day of the meeting (Chris Arnold, member of the Board of Directors). The roadmap sets the goals and targets for HVP activities and lays out strategies for their attainment (http://short.variome.org/roadmap). It is structured around four key themes or pillars that underpin all the activities of the HVP Consortium.

1. Setting normative function involves the work and activities centered on defining the standards, systems, and infrastructure across countries and diseases that are at the core of the HVP as well as the processes and best practices that govern their effective operation.

2. Behaving ethically ensures that everything is done in a manner that is consistent with the full range of ethical, legal, and social differences that are part of data sharing in a diverse world.

3. Sharing knowledge emphasizes sharing of the techniques and best practices for collection, curation, interpretation, and sharing of genetic variation data, providing public education initiatives and sharing of knowledge to advance the field of medical genetics and genomics.

4. Building capacity involves working with various stakeholders in countries and regions to increase capacity through education and skills training and to integrate the skills and knowledge gained into more effective practice.
The keynote lecture, "Translating variation into health," was given by Sir John Burn, who outlined the aspirations of the HVP to provide easy online access to all gene variants and their functional significance. He spoke about the complexity of interpreting the phenotype from the wide spectrum of genetic variation that is now being discovered by next-generation sequencing and advocated a combination of rewards and regulation to ensure timely and complete collection of data and a standard approach to assessing the probability of variant pathogenicity.

\section{SUMMARY OF SESSIONS}

The structure of the HVP4 was somewhat different from that of previous meetings, which focused largely on scientific and technical presentations. This meeting was structured around the four main themes of the roadmap and included extensive discussions by the members to formulate an action plan. Agreed actions were promulgated at the project's Advisory Council and Scientific Advisory Committee postconference meetings.

\section{BEHAVING ETHICALLY: PROJECTWIDE ETHICAL CONSIDERATIONS}

Behaving ethically is one of the four pillars of HVP activities outlined in Project Roadmap 2012-2016. Previously, the HVP Consortium has focused its activities in this area on the ethics of collecting and storing genetic variation information in databases. ${ }^{2,7}$ Building from the discussions of the HVP Beijing Meeting, ${ }^{6}$ the ethics session at the HVP4 took a much broader view of ethical behavior within the consortium.

The goal of the session was to generate discussion of a more nuanced approach to ethics across all the activities carried out by HVP Consortium members. The discussions drew on how the existing HVP core values (http://www.humanvariomeproject.org/index.php/about/vision) can remain current to both the aims of HVP and the practice of members, and whether the existing core values could be used to develop a code of conduct for the HVP Consortium. After much discussion, the delegates concluded that any discussion of such a code cannot be seen in isolation from other activities linked to sharing of data across the globe in a manner that is both nondiscriminatory and transparent.

Delegates were also tasked with beginning to develop a code of conduct that would encompass the agreed-upon core values and principles and determine specific obligations and rights. The scope of the code was considered as well as whether it ought to pertain solely to the behavior of Consortium members or be extended to include nonmembers contributing to member infrastructure (i.e., individuals submitting data to HVP country nodes). It was recognized that the HVP is not the only player in this field, and many existing groups working in various parts of the world already possess vast experience in dealing with these issues. These groups include ongoing scientific initiatives and organizations active in the field of ethical, legal, and social issues, e.g., UNESCO, the World Health Organization, and the Human Genome Organization. There is much expertise and 
experience to draw on, and it would serve both the project and society in general to ensure that all groups collaborate in an ongoing and participatory manner. It was also recognized that there are often competing views, interests, and priorities but that the HVP must ensure (i) that there is thorough understanding and open discussion of the issues and (ii) that policies and practices in the HVP are informed by various existing international declarations and agreements.

The session concluded with an agreement that the project's Ethics Interest Group would be asked to review the current code of conduct to incorporate the updated core values. The group would also determine a list of the other areas in which these issues need to be taken up in a more systematic way and suggest a work program to address them for discussion by the broader membership. The Ethics Interest Group was deemed a vital part of the HVP Consortium structure and a key asset in promoting a participatory, ongoing discussion and improved practice on the ethical and legal issues raised by the sharing of genetic and clinical information.

\section{BUILDING CAPACITY: HVP INITIATIVES IN EDUCATION AND SKILL DEVELOPMENT}

Education and skill development has been a prominent activity of HVP since the 2008 Planning Meeting. ${ }^{5}$ In 2009, the group was charged with conducting a needs assessment, and the findings were presented at an HVP forum in November 2010. The assessment found that the most frequently chosen category for unmet needs was methods to interpret variants. Other needs included submission and searching methods, the incorporation of genetics into clinical workflow, and criteria for variation/ phenotype associations.

This session used the findings of the needs assessment to frame a new discussion about education and training priorities across the field of medical genetics and genomics. Delegates recognized that skill shortages were being faced in both developed and developing countries for different reasons and expressed support for an active role for the HVP in addressing this issue in a more systematic way. Although most of these issues are currently handled at the national level because of the regulatory frameworks for credentials and qualifications, an international perspective on some issues may be useful, given the increasingly global nature of labor markets. Consequently, the Education and Training Interest Group is to expand its membership and take on a broader range of issues consistent with the priority areas outlined in Project Roadmap 2012-2016:

1. An internal HVP function with a focus on keeping consortium members up to date on most recent research outcomes and policy issues.

2. Focus on young people and how the HVP can encourage members to attract young people to the field.

3. Increase awareness of medical genetics and genomics among government officials (both politicians and policy makers), the general public, and the media.
4. Integrate skill-enhancement and capacity-building activities into other HVP activities, particularly those focused on building new HVP country nodes in low- and middleincome countries.

Delegates also recognized the need to be able to report results achieved in education and training activities. This monitoring and evaluation function is extremely important, and the HVP has a strong role to play in creating a strong evidence base for what works in capacity-building programs and sharing relevant best practices.

\section{SETTING NORMATIVE FUNCTION: DESCRIBING PHENOTYPE}

"Setting normative function" refers to the process of developing, communicating, and monitoring the implementation of standards, systems, and infrastructure for the key activities of the HVP. The ability to unambiguously describe phenotypes for all human disease in a manner that can be used and understood by both humans and computers is a challenge that cuts across the entire project. This session discussed the logistics of collection and reporting of improved phenotype data.

Gene/disease-specific databases must strive to include more "deep phenotype information" that describes a wider spectrum of disease features, possibly including biochemical findings and gene expression, depending on the purpose and scope of databases. In addition to the traditional goal of genotypephenotype correlations, an emerging need is to monitor the effects of medical interventions and natural history, but currently used phenotype ontologies/terminologies are not adequate. Therefore, this is a priority area for future development. Ontologies from related areas need to be harmonized to make different databases as interoperable as possible and to allow the use of multiple ontologies in one database (e.g., ontology for disease names, phenotypes, drugs, treatments). It would be useful to have agreement on a core set of about 1,500 terms for phenotype ontologies with standard definitions. Data entry will also need to be simplified to enable medical doctors to enter data on their patients over time (longitudinally) as well as at different levels of detail. For example, whole-genome data, if available, will need to be accessed and utilized throughout the patient's life as new medical problems emerge.

These discussions were continued at the satellite meeting of the American Society of Human Genetics, "The Human Phenome Project," in San Francisco, November 2012.

\section{SHARING KNOWLEDGE: HOW THE MODEL OF INSIGHT CAN INFORM OTHER GENE/DISEASE-SPECIFIC DATABASES}

The International Society of Gastrointestinal Hereditary Tumours (InSiGHT) is the peak organization representing clinicians and scientists working on inheritable gastrointestinal cancers. In 2007, a pilot program was established with the HVP to collect all inherited variation affecting colon cancer susceptibility genes (Finlay Macrae). Since then, multiple 
variant repositories have been merged to form the InSiGHT colon cancer gene variant databases on the Leiden Open Variation Database platform, currently comprising 11 genes (http://chromium.liacs.nl/LOVD2/colon_cancer/home.php). A governance committee monitors database security, back up, access, and confidentiality and also oversees several specialist database committees that work on the interpretation, functional assays, phenotype, and virtual pathology of the gene variants.

All database submitters are encouraged to include at least a minimum data set of the variant-associated phenotype. ${ }^{8,9}$ A significant proportion of the missense mutations of the mismatch repair genes (Lynch syndrome) in the database has discordant interpretations regarding pathogenicity. Assignment of pathogenicity for "variants of unknown significance" carries some risk of being inaccurate, which may lead to adverse health outcomes. This led to the decision by the InSiGHT Council to incorporate the organization, which enables pathogenicity to be assigned to variants with substantial medico-legal protection. The InSiGHT Pathogenicity Committee (chair, Maurizio Genuardi) has customized the five-tier system of the International Agency for Research on Cancer to help classify these variants of unknown significance. The committee comprises more than 30 active members, who independently evaluate all the available evidence in favor of or against pathogenicity for each set of variants and subsequently reach consensus in an international teleconference. In addition to published data, unpublished information is canvassed from the wider InSiGHT membership. One line entry for each variant is included in the InSiGHT database describing outcomes.

\section{SETTING NORMATIVE FUNCTION: IDENTIFICATION OF AREAS IN WHICH STANDARDS AND GUIDELINES ARE REQUIRED}

The HVP produces two categories of recommendations: standards and guidelines. HVP standards are those systems, procedures, and technologies that the consortium has decided to adopt, whereas guidelines are perceived as beneficial but less prescriptive. Standards and guidelines are central to supporting the work of the HVP Consortium and cover a wide range of fields and disciplines, from ethics to nomenclature, data transfer protocols to collection protocols from clinics. This session identified several areas in which HVP could fill existing technical gaps or evaluate and standardize existing options. New models need to be developed for the "ideal databases," possibly different models for each level of complexity, ranging from simple variants to whole-genome data. It is also important to identify the most suitable data formats for storage and transport between databases. An international, central data "clearinghouse" is required that can issue unique identifiers for patients and/or records. This could be expanded to become a service that handles the distribution of next-generation sequencing data to relevant gene/diseasespecific databases for curation and interpretation. Common variants should also be included in gene/disease-specific databases to provide a reference point for the clearly pathogenic variants. Standards are required for phenotype description and distinguishing between the continuum of phenotypes from normal variation to mild or severe disease as well as for including data from family members, i.e., affected, nonaffected, siblings. Describing ethnicity is important in databases but needs to be standardized to remove stigma and to protect privacy.

\section{SETTING NORMATIVE FUNCTION: ACTIVITY PROPOSAL DEVELOPMENT}

HVP standards and guidelines are developed through a common process that is triggered whenever a consortium member submits a request to the International Scientific Advisory Committee for review (http://www.humanvariomeproject.org/ index.php/recommendations). This session selected five areas identified in the previous session and explored them further for immediate action:

1. Work with biomedical journals to develop policies that mandate submission to database prior to publication (Raymond Dalgleish). The HVP should provide a list of preferred databases for submission and "name and shame" those journals that do not mandate submission. The HVP should not only focus on genetics journals but also work with medical journals.

2. Standardize database fields (Johan den Dunnen). This will provide a basis for a database accreditation program. Several existing guidelines/resources were recognized, such as BioDBcore, the minimum information about a biomedical/biological investigation community (MIAME, MIAPE), and Gen2Phen minimal locus-specific variation database content.

3. Pathogenicity determination (Marc Greenblatt). The HVP needs to develop a core set of different types of evidence that are common to all genes. Existing resources in this area were recognized, such as UniProt evidence codes, evidence code ontology, and VariO.

4. Curation as a profession (Mireille Claustres). The HVP should recommend a process for accreditation of curators and investigate whether the term should be changed to "clinical bioinformatician." Existing resources could be utilized, such as the BioCuration Society, position descriptions from organizations that employ curators (e.g., National Center for Biotechnology Information, European Bioinformatics Institute), and the Master of Medical Sciences in Biomedical Informatics Program at Harvard Medical School.

5. Ethics and legal issues (Maria Jesus Sobrido). The HVP should ensure that members have legal protection to publish genetic data in databases and work with UNESCO and the World Health Organization to develop a resolution encouraging member states to facilitate data sharing. 


\section{SETTING NORMATIVE FUNCTION: ACCREDITATION OF GENE/DISEASE-SPECIFIC DATABASES}

The delegates agreed unanimously that accreditation of databases is required. However, there are no established quality schemes in place, and only one database (Diagnostic Mutation Database) has had a quality assessment (by The European Molecular Genetics Quality Network). Therefore, evaluation criteria need to be developed. Some of the assessment can be based on self-assessment-a web questionnaire to be filled in by curators. In the case of databases based on the Leiden Open Variation Database model, these details can be automatically collected. However, there will still be a number of points to be assessed manually or in another way, and assessors need to be impartial. The possibility of subcontracting was discussed.

Databases should publish statistics on the number of submitters, their geographic distribution, and similar metrics. User feedback should be utilized in accreditation ("did these data help your patient?" - an eBay-like feedback system), and users should be able to flag inaccurate data. It was recognized that removing all errors from a database would be impossible. This highlights the need for the use of evidence codes, tracking data provenance, etc. Perhaps separate accreditation systems are required for the database and the data. A resolution on who would do the evaluation was not reached. A working group was agreed on, with Andrew Devereau, John Hancock, Peter Taschner, and Mauno Vihinen as the initial members.

\section{HVP COUNTRY NODES AND THEIR FUTURE DEVELOPMENT}

The last day of the meeting focused on the role and functions of HVP Country Nodes and their future development. An important part of the day was the sharing of knowledge and experience from different parts of the world and to hear the richness and diversity of practice, especially from countries often thought of as having fewer resources.

Given the diversity and range of practice across the formally recognized HVP Country Nodes as well as those nodes that are in the process of formalizing their activities, a key issue is just what constitutes an HVP Country Node. It is well understood that "one size does not fit all" and that nodes must respond to their local conditions to function effectively. At the national level, countries differ in their levels of political support, regulatory environments, availability of financial and human resources, as well as the cultural and religious diversity of their communities. This diversity can be a double-edged sword; although it allows for a variety of practices, it can also slow the processes of establishing nodes and sharing data because there are too many choices that can be made early in the process.

In an attempt to address these issues and discuss them in an open and transparent manner, the day began with a presentation by the International Coordinating Office of a possible definition of an HVP Country Node. The article on which this was based had been circulated in advance as a "conversation starter" (see http://short.variome.org/PP-ICO-05-2012). It presented country nodes as evolving entities, moving from an early or preformal stage called "getting ready," through four stages of increasing formality, until the country node could be recognized as being "fully functional." Each of these stages was defined using the following five areas of activity considered to be core to a well-functioning node: data collection and sharing; having the necessary resources to carry out activities; the existence of ethical, legal, and social frameworks required to operate effectively in local environment; linkages to the national health system; and engagement in debate of medical genetics and genomics issues at the national level.

Discussion was then taken up in three sessions-HVP country and regional nodes: toward a definition, HVP country and regional nodes: exploring a definition, and a process for accreditation of HVP country nodes.

Following a series of lively discussions among the three groups of panelists and the many questions drawn from the audience, it was agreed that although there was a need to attempt to define HVP Country Nodes more clearly, it was too early to agree because this would risk stifling the growing level of activity at the country level. It was also agreed that issues of quality of practice, including accreditation, were very important and that it was necessary to have these on the table to guide future developments.

It was clear that the HVP's International Confederation of Countries Advisory Council has a role in guiding further discussion of these issues and ensuring that:

- Information on current practice of HVP Country Nodes is collected and shared in a more systematic manner and is part of knowledge sharing and capacity building.

- Information on the development of HVP Country Nodes into more formal entities and how they collect and share data need to be effectively monitored and reported.

- Data collection and sharing aspects should be the priority activity for the next 2 years.

- HVP Country Nodes need to engage in ethical, legal, and social issues and capacity-building activities of the HVP, as discussed during the first day of the meeting.

\section{LOOKING TO THE FUTURE}

In the final session of the meeting, delegates were treated to two expert overviews focusing on the theme of "Genomics and World Health." Sir David Weatherall, Weatherall Institute of Molecular Medicine, University of Oxford, addressed the challenges of translating advances in genetics and genomics into health care. By tracing the progress made in the genomics and control of hemoglobin disorders, the third most common group of birth defects worldwide, vascular diseases, communicable diseases, and pharmocogenomics, he highlighted the rapid advances in research at the molecular level in recent years. He pointed to the important advances made through collaborations between countries and institutions in north-south and south-south networks. He said that medical 
education, particularly, in high-income countries had been very slow to include the field in their curriculum and that this issue would be increasingly important in translating advances in research into medical practice. The opportunity to find more cost-effective ways of treating common diseases in highincome countries, which already have pressures on their health budgets, will be increasingly important for fulfilling the promise of human genetics and genomics. Sir David challenged the HVP Consortium members to engage in the global debates on these issues and to ensure that there was also an informed and open discussion in their own countries.

Myles Axton, editor of Nature Genetics, also took on the future contribution of human genetics and genomics in a world of global disparities in health outcomes in his address. He suggested that the advances in research in recent years saw us close to understanding the convergence of Mendelian concepts with those of natural variations in gene transcriptions. He echoed Sir David Weatherall's thoughts by also stressing that the three branches of medicine-research, clinical delivery, and quality/cost assurances-needed to embrace the gains made in the field of human genetics and genomics in a more systematic way in future years. He raised the importance of the role of patients in these processes-the vital need to ensure that they are seen as true partners. They are much more than partners in the research process, as they are active players in raising funding, advocacy with government, and voicing their needs to the broader society.

In addressing the future, Dr Axton highlighted the role that data sharing will have in delivering advances in health care to patients and communities. Only by drawing together efforts, population by population, in culturally sensitive ways and by leveraging modern technologies to share knowledge will better outcomes be realized.

\section{CONCLUSION}

The 4th Biennial Meeting of the HVP Consortium provided a platform for the members to participate and discuss the actions required to further the aims of the project over the next 2 to 5 years. Dr Axton summed it up very well when he said that only by taking a global view of data collection and knowledge sharing across diseases and countries will the results to patients be delivered in efficient and effective ways. The commitment to this endeavor was clearly demonstrated at this meeting.

\section{DISCLOSURE}

The authors declare no conflict of interest.

\section{REFERENCES}

1. Collins FS, Green ED, Guttmacher AE, Guyer MS. A vision for the future of genomics research. Nature 2003;422:835-847.

2. Kohonen-Corish MR, Al-Aama JY, Auerbach AD, et al. How to catch all those mutations-the report of the third Human Variome Project Meeting, UNESCO Paris, May 2010. Hum Mutat 2010;31:1374-1381.

3. Cotton RG, Appelbe W, Auerbach AD, et al. Recommendations of the 2006 Human Variome Project meeting. Nat Genet 2007;39:433-436.

4. Ring HZ, Kwok PY, Cotton RG. Human Variome Project: an international collaboration to catalogue human genetic variation. Pharmacogenomics 2006;7:969-972.

5. Kaput J, Cotton RG, Hardman L, et al. Planning the human variome project: the Spain report. Hum Mutat 2009;30:496-510.

6. Smith TD, Robinson HM, Cotton RG. The Human Variome Project Beijing meeting. J Med Genet 2012;49:284-289.

7. Povey S, Al Aqeel Al, Cambon-Thomsen A, et al. Practical guidelines addressing ethical issues pertaining to the curation of human locus-specific variation databases (LSDBs). Hum Mutat 2010;31:1179-1184.

8. Kohonen-Corish M, Weber TK, Lindblom A, Macrae F; Meeting Participants. Report of the combined meeting of the International Society for Gastrointestinal Hereditary Tumours, the Human Variome Project and the National Cancer Institute Colon Cancer Family Registry, Duesseldorf, Germany, 24 June 2009. Fam Cancer 2010;9:705-711.

9. Kohonen-Corish MR, Macrae F, Genuardi M, et al.; Contributors to the InSiGHT-HVP Workshop. Deciphering the colon cancer genes-report of the InSiGHT-Human Variome Project Workshop, UNESCO, Paris 2010. Hum Mutat 2011;32:491-494. 